\title{
Stereoselective Renal Clearance of Pindolol in Humans
}

Poe-Hirr Hsyu and Kathleen M. Giacomini

School of Pharmacy, University of California, San Francisco, California 94143

\begin{abstract}
In this study, pindolol, a $\boldsymbol{\beta}$-adrenoceptor blocking agent marketed as a racemic mixture, was used as a model compound to investigate stereoselective renal clearance of organic cations in human beings. Six normal subjects received an oral dose of $20 \mathrm{mg}$ racemic pindolol. Heart rate and blood pressure were measured throughout the study. A stereospecific high performance liquid chromatographic procedure was used to quantitate the concentrations of $d$-and $l$-pindolol in plasma and urine. Renal clearance and other pharmacokinetic parameters of both enantiomers were calculated and compared. The renal clearance of $l$-pindolol was greater than that of $d$-pindolol in all subjects. The renal clearance (mean \pm SD) was $240 \pm 55 \mathrm{ml} / \mathrm{min}$ for $l$-pindolol and $200 \pm 51 \mathrm{ml} /$ min for $d$-pindolol $(P<0.01)$. Since stereoselective binding to plasma proteins was not observed, differences in renal clearance between $d$ - and $l$-pindolol were caused by either stereoselective renal transport, or stereoselective renal metabolism. The area under the plasma concentration-time curve, the amount of drug excreted, and the half-life of $l$-pindolol were greater than those of $d$-pindolol, which suggests that pindolol was also eliminated stereoselectively by nonrenal routes. The slopes of the resting heart rate vs. the plasma concentration of $l$-pindolol were significantly less than zero and were significantly correlated to the pretreatment heart rate, which supports the hypothesis that intrinsic sympathetic tone largely determines the effect of pindolol on the resting heart rate. The observation that pindolol is eliminated stereoselectively by the kidney may have clinical implications for other racemic drugs that are renally eliminated.
\end{abstract}

\section{Introduction}

A number of drugs are marketed as racemic mixtures. Although enantiomers have identical physical and chemical properties, the chiral macromolecules, especially proteins, in the body are very specific to the spatial arrangement of drug molecules. Consequently, stereospecific or stereoselective interactions between macromolecules and drugs are frequently encountered. These interactions result in stereoselective pharmacokinetics and pharmacodynamics of drugs. For example, $S$-(-)-propranolol is a considerably more potent $\beta$-adrenoceptor blocking agent than the $R$-enantiomer (1). In addition, the clearance of propranolol in human beings is stereoselective, with the $\mathrm{S}$-enantiomer cleared less rapidly than the $R$-enantiomer $(2,3)$.

Address reprint requests to Dr. Giacomini, Room 926-S, School of Pharmacy, University of California, San Francisco, CA 94143. 1985 .

Received for publication 24 May 1985 and in revised form 30 July

J. Clin. Invest.

(C) The American Society for Clinical Investigation, Inc.

0021-9738/85/11/1720/07 \$1.00

Volume 76, November 1985, 1720-1726
Stereoselective hepatic metabolism of drugs has been well documented both in vitro and in vivo (4). The clinical implications and complexities of stereoselective metabolism are exemplified by the interaction of warfarin and phenylbutazone (5). The clearance of $S$-warfarin, the more potent enantiomer, is reduced approximately to one-half by coadministration of phenylbutazone, whereas the clearance of $R$-warfarin is almost doubled. The measured clearance of the racemate, however, is unaffected. Thus, in the presence of phenylbutazone, plasma concentrations of racemic warfarin remain unchanged, whereas the anticoagulant effect of warfarin is augmented.

In contrast to the numerous studies regarding stereoselective hepatic metabolism of drugs, few studies have determined whether stereoselective renal excretion of drugs occurs. Renal excretion is a major route of elimination of drugs and their metabolites and is composed of three processes: glomerular filtration, tubular secretion, and tubular reabsorption. Of these, tubular secretion and reabsorption, which can involve saturable carrier-mediated processes, may be stereoselective. For example, the transport of endogenous compounds such as amino acids in the proximal tubule is stereoselective (6). Two systems are primarily responsible for the active secretion of drugs from the body: one for organic anions and another for organic cations. At present, stereoselective renal elimination of either cationic or anionic drugs has been rarely studied.

Pindolol (Visken Sandoz Inc., East Hanover, NJ), an organic base, is a clinically important $\beta$-adrenoceptor blocking agent. In humans, the drug is cleared approximately equally by both metabolism and renal excretion (7). The renal clearance of racemic pindolol exceeds the glomerular filtration rate (GFR), ${ }^{1}$ which indicates that the compound is actively secreted. In this study, we examined the stereoselective pharmacokinetics and pharmacodynamics of pindolol in humans. Particular emphasis was placed on measuring the renal clearance of the individual enantiomers. A stereospecific high performance liquid chromatographic assay for pindolol in biological fluids developed in our laboratory enabled us to quantify the concentrations of both enantiomers simultaneously in plasma and in urine. Thus, we were able to measure the renal clearance of each enantiomer in the presence of the other enantiomer under identical physiological conditions.

Our results indicate that the renal clearance of pindolol is stereoselective in humans. The mechanisms that may be responsible for the observed stereoselective renal clearance are discussed.

\section{Methods}

Clinical protocol. Six healthy male volunteers between 20 and $30 \mathrm{yr}$ of age, weighing between 60 and $80 \mathrm{~kg}$, were studied. None had a history

1. Abbreviations used in this paper: AUC, area under the plasma-concentration curve; $\mathrm{Ae}^{\infty}$, amount of unchanged drug excreted in the urine from time 0 to time infinity; fu, fraction unbound of drugs in plasma; GFR, glomerular filtration rate. 
of diabetes, asthma, or other respiratory, hepatic, renal, or cardiovascular disease. Informed consent was obtained after the purpose, procedures, and risks of the experiments were explained to the volunteers. The volunteers were instructed not to take any drugs or alcoholic beverages for $2 \mathrm{wk}$ before the experiments. After an overnight fast, an indwelling polyethylene venous catheter with a Teflon obturator (20G2 Longdwel obturator; Becton-Dickinson, Rutherford, NJ) was placed in a forearm vein of each subject. Subjects swallowed two $10-\mathrm{mg}$ tablets of pindolol (Visken) along with $240 \mathrm{ml}$ of lukewarm water. Blood samples were collected through the catheter into rubber-sealed glass test tubes containing heparin as an anticoagulant (10-ml Vacutainer heparin tube; Becton-Dickinson). No heparin was administered to the subjects. $40 \mathrm{ml}$ of blood was collected before drug administration and $10 \mathrm{ml}$ of blood was collected at $\sim 0.5,0.75,1,1.5,2,3,4,6,8,10,12$, and $24 \mathrm{~h}$ after drug administration. Blood was centrifuged immediately at $1,000 \mathrm{~g}$ and the plasma was transferred into screw-topped glass vials. Urine was collected into plastic graduated containers at intervals of $0-0.5,0.5-1$, $1-2,2-3,3-4,4-6,6-8,8-12$, and $12-24 \mathrm{~h}$ after drug administration. To facilitate urination, the subjects drank an additional $240 \mathrm{ml}$ of lukewarm water at the end of each of the first three urine collection intervals. Immediately after collection, the $\mathrm{pH}$ and volume of the urine were measured and recorded, and a $20-\mathrm{ml}$ aliquot was saved in a screw-capped, glass scintillation counting vial. Plasma and urine were stored at $-20^{\circ} \mathrm{C}$ until analysis.

Blood pressure and heart rate were measured in subjects in the supine position immediately before the collection of each blood sample. Blood pressure measurements were determined by a sphygmomanometer and expressed as mean arterial pressure. The heart rate was obtained from an electrocardiogram (1511B electrocardiograph, four-lead; HewlettPackard Co., Palo Alto, CA).

The experiments were carried out in the Drug Studies Unit of the University of California, San Francisco.

Analytical. Concentrations of $d$ - and $l$-pindolol in plasma and urine were determined by a stereospecific high performance liquid chromatographic procedure (Hsyu, P.-H., and K. M. Giacomini, manuscript submitted for publication). Briefly, the procedure involved extraction into ether of $d$ - and $l$-pindolol from alkalinized (with $1 \mathrm{~N} \mathrm{NaOH}$ ) biological fluids. Samples were further purified by two steps of back-extraction. The ether layer was then dried under nitrogen stream and reacted at room temperature with $S-(-)-\alpha$-methylbenzyl isocyanate in chloroform to form diastereomers of pindolol. Subsequently, the chloroform was evaporated under a nitrogen stream and the diastereomers were reconstituted in $100 \mu$ l of mobile phase (methanol/water, 62:38). The solution was injected onto a high performance liquid chromatographic system equipped with a pump (110A; Beckman Instruments, Inc., Fullerton, CA), an injector (U6K; Waters Associates, Millipore Corp., Milford, MA), and a variable-wavelength fluorescence detector (FS 970 fluorometer and GM 970 monochromator; Kratos Analytical Instruments, Ramsey, NJ). The column was a reversed-phase C-18 column (Altex Ultrasphere ODS $5 \mu \mathrm{m}, 4.6 \mathrm{~mm} \times 15 \mathrm{~cm}$; Beckman Instruments, Inc., Berkeley, CA). The flow of the mobile phase was $1.2 \mathrm{ml} / \mathrm{min}$ at a column inlet pressure of $\sim 1,900 \mathrm{psi}$. The excitation wavelength of fluorescence was set at $209 \mathrm{~nm}$ and an emission filter was present that excluded light having wavelengths of $<320 \mathrm{~nm}$. Under these conditions, base-line resolution of the diastereomers was obtained with retention times of 13.8 and $15.6 \mathrm{~min}$ for the $d$ - and $l$-pindolol diastereomers, respectively. Concentrations of $d$ - and $l$-pindolol in each plasma and urine sample were determined in duplicate using calibration curves constructed by analyzing known quantities of $d$ - and $l$-pindolol in the plasma and urine. The calibration curves were linear $(r>0.999)$ and reproducible (coefficient of variation typically $<12 \%$ ). The detection limit of the assay, defined as four times the base-line noise, was $2 \mathrm{ng} / \mathrm{ml}$ for both enantiomers of pindolol in plasma.

Creatinine concentrations in the plasma and urine were determined by a creatinine diagnostic kit (Sigma Chemical Co., St. Louis, MO), in which the colorimetric reaction between creatinine and picric acid was used to quantitate the creatinine concentration.
Plasma protein binding. The binding of $d$ - and $l$-pindolol to plasma proteins was determined by equilibrium dialysis. 2-ml dialysis cells (Dianorm System, Spectrum Medical, Los Angeles, CA), separated into two compartments by a cellophane membrane (Spectra/Por II, Spectrum Medical) with a molecular weight cutoff of $12,000-14,000$, were used. The dialysis was carried out at $37^{\circ} \mathrm{C}$ for $4 \mathrm{~h}$, during which time the cells were gently rotated. In preliminary studies, the 4-h dialysis time was found to be sufficiently long to achieve equilibrium (greater than five times the time required to reach $50 \%$ of equilibrium value). The effect of concentration on the binding of pindolol to plasma proteins was also examined in the preliminary studies. The results indicated that the fraction unbound ( $\mathrm{fu}$ ) of both enantiomers of pindolol was constant when the concentrations of racemic pindolol were $<1.5 \mu \mathrm{g} / \mathrm{ml}$. Therefore, $800 \mathrm{ng}$ of racemic pindolol was added to $1 \mathrm{ml}$ of blank plasma from each subject and dialyzed against $1 \mathrm{ml}$ of isotonic Sorensen's phosphate buffer $(0.13$ $\mathrm{M}, \mathrm{pH}$ 7.4) in triplicate. After dialysis, the concentrations of $l$ - and $d$ pindolol in the plasma and in the buffer were determined by the stereospecific high performance liquid chromatographic procedure described in the preceding section. The fu was calculated as the concentration of each enantiomer in the buffer divided by the concentration of the same enantiomer in the plasma.

Data analysis. Because the plasma concentrations of $d$-and $l$-pindolol at $24 \mathrm{~h}$ in each subject were below the detection limit of the high performance liquid chromatographic procedure, the area under the plasma concentration vs. time curve (AUC) of the individual enantiomers of pindolol was calculated by the linear trapezoidal rule from time 0 to 12 $\mathrm{h}$ and extrapolated to time infinity. The extrapolated area under the curve from $12 \mathrm{~h}$ to time infinity, $\mathrm{AUC}_{\text {last }-\infty}$, was obtained by the formula $\mathrm{AUC}_{\text {last } \rightarrow \infty}=C_{\text {last }} / K$, where $C_{\text {last }}$ is the last measured concentration of the enantiomers (usually at $12 \mathrm{~h}$ ) and $K$ is the terminal elimination rate constant obtained from curve fitting. In each subject, the plasma concentration-time curve of each enantiomer was fit to a one-compartment model with zero-order absorption using the DRUGFUN program from the PROPHET system (8). Zero-order rather than first-order absorption was used to fit the plasma concentration-time curves because a better fit as judged by the Schwartz criterion (9) was obtained. The mechanisms involved in the absorption of pindolol were not studied and should not be implied by the fitting procedure. The terminal half-life of the individual pindolol enantiomers in each subject was obtained from the fits. To obtain the renal clearance of each enantiomer, the excretion rate was plotted against the plasma concentration obtained at the midpoint of each urine collection interval and a linear least-squares regression analysis forced through the origin was applied. The slope of this line is the renal clearance (10). Renal clearance was also calculated as $\mathrm{Ae}^{\infty} / \mathrm{AUC}$, where $\mathrm{Ae}^{\infty}$ is the amount of unchanged pindolol enantiomer excreted in the urine from time 0 to time infinity.

To ascertain whether renal clearance was dependent upon urine $\mathrm{pH}$, urine flow rate, time, or plasma concentration, fractional renal clearances were calculated for each urine collection period as the excretion rate divided by the measured plasma concentration obtained at the midpoint of the urine collection intervals. Plots of fractional renal clearance vs. urine $\mathrm{pH}$, urine flow rate, time, and plasma concentration (obtained at the midpoint of the urine collection period) were examined visually and also by a linear least-squares regression analysis for systematic trends.

The GFR in each subject was estimated from the creatinine clearance calculated as the excretion rate of creatinine divided by the plasma concentration of creatinine.

A statistical analysis to determine whether there were differences between $d$ - and $l$-pindolol in the individual pharmacokinetic parameters was carried out by the Student's paired $t$ test.

A pharmacodynamic analysis was carried out as follows. The heart rate and mean arterial pressure were plotted against the plasma concentration of $l$-pindolol, the active enantiomer (11), and linear regression was performed. To test whether a pharmacologic measurement in an individual subject was significantly related to the plasma concentration, the slope of each regression line was compared to zero by the Student's $t$ test. 

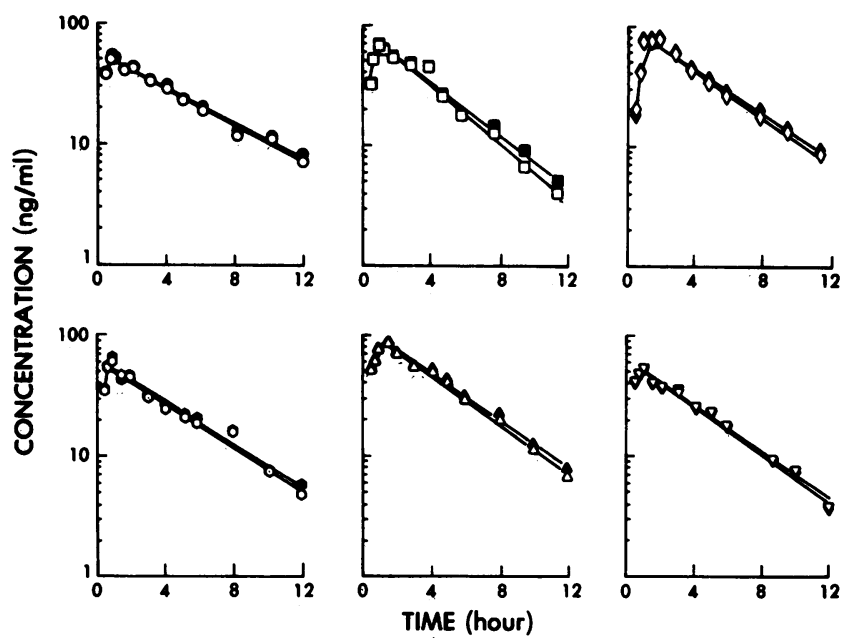

Figure 1. Plasma concentration-time profiles of $l$ - and $d$-pindolol in each subject after administration of an oral dose of $20 \mathrm{mg}$ of racemic pindolol. The curves represent the best fit with a one-compartment model with an absorption phase. Each symbol represents an individual subject: K.C. (circles); F.W. (squares); K.D. (diamonds); P.E. (hexagons); B.D. (triangles); M.P. (inverted triangles). Solid symbols represent $l$-pindolol and open symbols represent $d$-pindolol. The designation of symbols is identical in all figures.

\section{Results}

Plasma concentration-time profiles of both enantiomers in all subjects are presented in Fig. 1. The data can be described by a one-compartment model with an absorption phase. In each subject, the maximum concentrations of $d$ - and $l$-pindolol occurred at the same time (between $\mathrm{l}$ and $2 \mathrm{~h}$ after drug administration). In addition, the maximum concentrations of $d$ - and $l$-pindolol were identical in each subject and averaged $66 \pm 13 \mathrm{ng} / \mathrm{ml}$ (mean $\pm \mathrm{SD}$ ) in the six subjects. However, the plasma concentration of $d$ - and $l$-pindolol tended to diverge at later times, with concentrations of $l$-pindolol being slightly higher than $d$-pindolol in five of the six subjects.

The pharmacokinetic parameters of $d$ - and $l$-pindolol are summarized in Table I. The AUC and the terminal half-life of $l$-pindolol were greater than those of $d$-pindolol $(P<0.05)$. In all subjects, the amount of $l$-pindolol excreted unchanged in the urine during the experiment $\left(\mathrm{Ae}^{\infty}\right)$ was greater than that of $d$ pindolol. Since the experiment was carried out for five to six half-lives of $d$ - or $l$-pindolol, these amounts approximated the total amount of the enantiomer that would be excreted. Of the 10 -mg dose of each enantiomer, the fraction of $l$-pindolol excreted in the urine was $0.430 \pm 0.067$ in the six subjects and was $0.361 \pm 0.074$ for $d$-pindolol.

Fig. 2 depicts plots of the renal excretion rate vs. the plasma concentration of the individual enantiomers in each of the six subjects. In several but not all subjects, there was a tendency for the renal clearance to be greater with higher plasma concentrations. The slopes of these plots represent the renal clearance of $d$ - and $l$-pindolol and are graphically presented in Fig. 3. The mean renal clearance of $l$-pindolol was $240 \pm 55 \mathrm{ml} / \mathrm{min}$ and was statistically greater than the value of $200 \pm 51 \mathrm{ml} / \mathrm{min}$ for $d$-pindolol $(P<0.01)$. When renal clearance was calculated as $\mathrm{Ae}^{\infty} /$ AUC, similar stereoselective differences were observed (176 \pm 55 $\mathrm{ml} / \mathrm{min}$ for $d$-pindolol, $204 \pm 61 \mathrm{ml} / \mathrm{min}$ for l-pindolol; $P$ $<0.005)$. The net clearance by secretion, calculated as renal clearance $-f u \times G F R$, is also shown in Fig. 3. The net clearance by secretion of $l$-pindolol was $196 \pm 47 \mathrm{ml} / \mathrm{min}$ and was $\sim 30 \%$ higher on the average than that of $d$-pindolol $(157 \pm 48 \mathrm{ml} / \mathrm{min}$; $P<0.01$ ).

Plots of renal clearance vs. urine $\mathrm{pH}$, time, and plasma concentration in each subject demonstrated no systematic trends, which suggests no dependence of renal clearance on these factors. When linear regression analysis was applied, the slopes of the plots of renal clearance vs. time or plasma concentration in five of the six subjects were not significantly different from zero $(P>0.05)$. With regard to urine $\mathrm{pH}$, none of the slopes was significantly different from zero. However, in three of the six subjects, the slope of renal clearance of either $d$ - or $l$-pindolol vs. urine flow was significantly different from zero $(P<0.05)$.

The fu of $d$ - and $l$-pindolol in each of the six subjects is shown in Table II. There were no significant differences in the fu of $d$ - and $l$-pindolol that averaged $\sim 0.45$. The renal clearances of unbound enantiomer, calculated as renal clearance divided by fu, are also shown in Table II together with the GFR. The unbound renal clearance of both enantiomers was approximately fivefold greater than the GFR, which demonstrates the existence of an active secretory process.

Table I. Pharmacokinetic Parameters of d-Pindolol and l-Pindolol in Humans

\begin{tabular}{|c|c|c|c|c|c|c|}
\hline \multirow[b]{2}{*}{ Subject } & \multicolumn{2}{|l|}{ AUC } & \multicolumn{2}{|l|}{ Half-life } & \multicolumn{2}{|l|}{$\mathrm{Ae}^{\infty}$} \\
\hline & $d$-Pindolol & l-Pindolol & $d$-Pindolol & l-Pindolol & $d$-Pindolol & l-Pindolol \\
\hline & $n g / m l \cdot h$ & $n g / m l \cdot h$ & $h$ & $h$ & $m g$ & $m g$ \\
\hline K.C. & 310 & 321 & 4.04 & 4.22 & 3.20 & 3.99 \\
\hline F.W. & 347 & 364 & 2.73 & 3.02 & 4.78 & 5.41 \\
\hline K.D. & 462 & 477 & 3.37 & 3.57 & 3.64 & 4.15 \\
\hline P.E. & 299 & 304 & 3.38 & 3.50 & 3.57 & 4.19 \\
\hline B.D. & 453 & 477 & 2.93 & 3.12 & 2.56 & 3.43 \\
\hline M.P. & 283 & 279 & 3.12 & 3.03 & 3.92 & 4.62 \\
\hline Mean & 359 & $370^{*}$ & 3.26 & $3.41^{*}$ & 3.61 & $4.30^{*}$ \\
\hline SD & 79 & 87 & 0.46 & 0.46 & 0.74 & 0.67 \\
\hline
\end{tabular}

\footnotetext{
* The difference between $d$ - and l-pindolol is statistically significant $(P<0.05)$ by Student's paired $t$ test.
} 


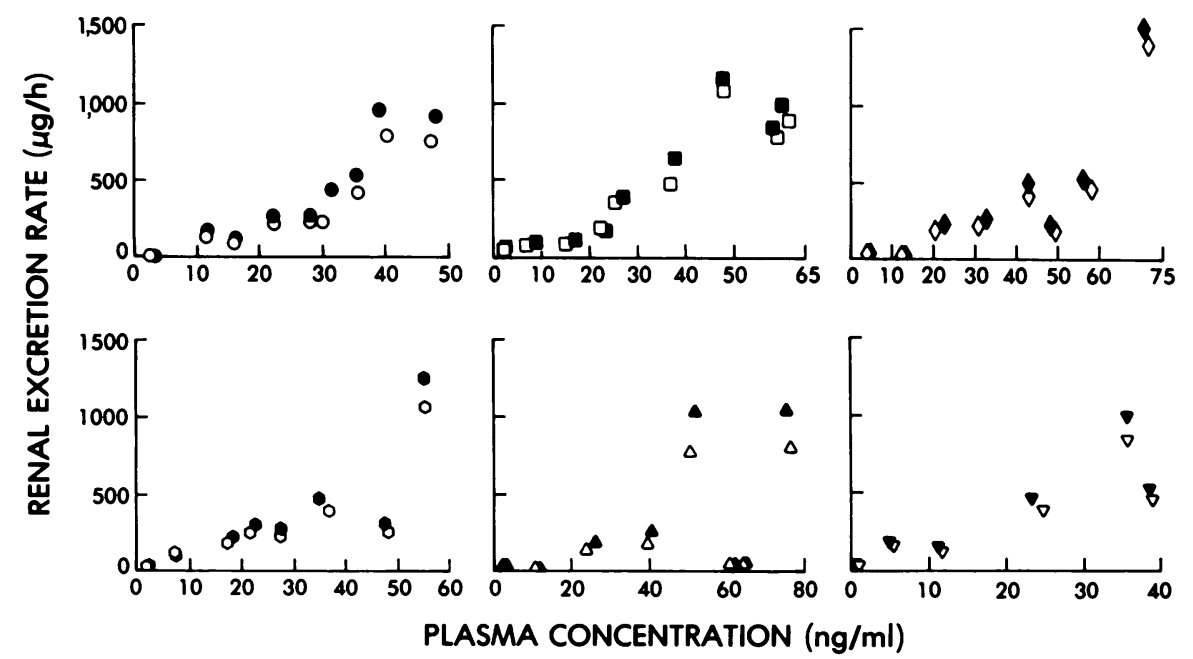

Figure 2. Renal excretion rate vs. plasma concentration (obtained at the midpoint of the urine collection interval) of $l$-pindolol (solid symbols) and $d$-pindolol (open symbols) in each subject.
Fig. 4 shows the plots of heart rate vs. plasma concentration of pindolol in each subject. A negative slope was obtained in all six subjects, which indicates that the heart rate was significantly reduced after a single oral dose of pindolol. The slope was significantly different from zero $(P<0.05)$ in four of the six subjects. Fig. 5 is a plot of the individual slopes vs. the initial heart rates. The correlation coefficient $(r)$ was $-0.917(P<0.01)$, which suggests that the greater the initial heart rate, the more prominent the effect of pindolol.

The mean arterial blood pressure was not affected by pindolol in three of the six subjects, as demonstrated by the fact that the slopes were not significantly different from zero. In two subjects, the slope of the mean arterial blood pressure vs. the plasma concentration was significantly negative $(P<0.05)$, and in one subject, the slope was significantly positive $(P<0.05)$.

\section{Discussion}

Although much has been learned in the past decade about stereoselective metabolism of drugs, little is known about stereoselective renal excretion. In this study, we used pindolol as a model compound to study whether stereoselective renal clearance of an organic base can be demonstrated clinically. Pindolol was ideally suited as a model compound for the following reasons. First, clinical experiments with racemic pindolol (12) have demonstrated that the drug is actively secreted in the kidneys. Second, a stereospecific high performance liquid chromatographic procedure developed in our laboratory enabled us to determine the concentration of each enantiomer in the presence of the other and thus to study the renal clearance of the individual enantiomers under identical physiological conditions. In addi- tion, because racemic pindolol is the clinically used compound, determining the renal clearance of the individual enantiomers after administration of the racemate is more relevant to therapy with pindolol.

The results of the present study indicate that pindolol is eliminated from the kidney stereoselectively (Figs. 2 and 3). Since each enantiomer was excreted from the kidney under identical physiological conditions, differences in factors such as urine $\mathrm{pH}$ or time cannot explain the observed stereoselective renal clearance.

There are three possible mechanisms that can account for the stereoselective renal clearance of pindolol: $(a)$ stereoselective plasma protein binding, $(b)$ stereoselective renal transport, and (c) stereoselective renal metabolism. Although pindolol was bound to plasma proteins with a fraction bound of $\sim 0.55$, no stereoselective differences in the binding of the individual enantiomers were observed (Table II). Thus, plasma protein binding cannot explain the observed stereoselective renal clearance of pindolol.

In this study, the renal clearance of each enantiomer was measured conventionally from the rate of excretion of unchanged enantiomer and the plasma concentration rather than from the renal blood flow and the extraction ratio of the enantiomer across the kidney. Thus, the renal clearance actually reflects the net excretion of unchanged enantiomer and not the total renal elimination (excretion plus metabolism). Renal metabolism represents a pathway that competes with the excretion of unchanged drug and, if present, diminishes the renal clearance of the enantiomer as calculated by the conventional method. Therefore, stereoselective differences in the metabolism of pindolol by the kidney could have produced the observed stereoselective renal
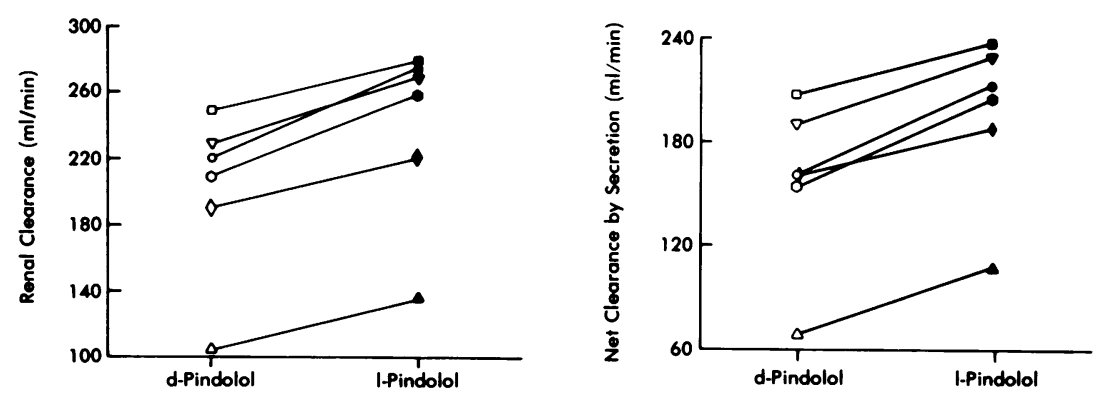

Figure 3. Renal clearance (left) and net clearance by secretion (right) of $l$-and $d$-pindolol in each subject. Net clearance by secretion was calculated as renal clearance minus fu $\times$ GFR. 
Table II. Protein Binding and Renal

Excretion of d-Pindolol and l-Pindolol

\begin{tabular}{|c|c|c|c|c|c|}
\hline \multirow[b]{2}{*}{ Subject } & \multicolumn{2}{|l|}{$\mathrm{fu}^{*}$} & \multicolumn{2}{|c|}{ Unbound renal clearance } & \multirow[b]{2}{*}{ GFR } \\
\hline & $d$-Pindolol & l-Pindolol & $d$-Pindolol & l-Pindolol & \\
\hline & & & $\mathrm{ml} / \mathrm{min}$ & $\mathrm{ml} / \mathrm{min}$ & $\mathrm{ml} / \mathrm{min}$ \\
\hline K.C. & 0.56 & 0.59 & 395 & 469 & 107 \\
\hline F.W. & 0.46 & 0.45 & 546 & 618 & 91 \\
\hline K.D. & 0.40 & 0.44 & 479 & 498 & 75 \\
\hline P.E. & 0.54 & 0.53 & 386 & 485 & 102 \\
\hline B.D. & 0.43 & 0.35 & 243 & 386 & 84 \\
\hline M.P. & 0.34 & 0.36 & 670 & 748 & 114 \\
\hline Mean & 0.45 & $0.45 \ddagger$ & 453 & $534 \S$ & 96 \\
\hline SD & 0.08 & 0.09 & 147 & 128 & 15 \\
\hline
\end{tabular}

* Each value represents mean of three replicate measurements. $\ddagger$ Not statistically different by paired $t$ test $(P>0.05)$.

$\S$ Statistically different by paired $t$ test $(P<0.01)$.

clearance of pindolol. However, several studies in the literature suggest that renal metabolism of pindolol in humans may not occur. In patients with renal disease, no change in the metabolic clearance of pindolol was observed $(13,14)$. If metabolism in the kidney were an important route of elimination, one might expect that the metabolic clearance of pindolol would be impaired in the diseased kidneys. In patients with liver disease, a substantial decrease in the clearance of racemic pindolol has been observed, which suggests that the metabolism of pindolol is occurring to a large extent in the liver $(15,16)$. If metabolism by the kidneys were an important pathway of elimination, only a small decrease in the clearance of pindolol in patients with liver disease would have been observed. Although none of these studies excludes the possibility that stereoselective renal metabolism of pindolol occurs in humans, the studies suggest that if renal metabolism does occur, it is a very minor route of elimination. However, it is possible that stereoselective renal metabolism, although a minor route, may account for the differences in the renal clearances of $d$ - and $l$-pindolol. If this were the case, $d$-pindolol would be cleared faster by renal metabolism than $l$ pindolol, resulting in a lower measured renal clearance of the $d$-enantiomer. This hypothesis is consistent with other data in this study that suggest that $d$-pindolol is cleared by nonrenal metabolism more quickly than $l$-pindolol (see below).

Another mechanism that may have been responsible for the stereoselective renal clearance of pindolol is stereoselective renal transport. In agreement with other studies $(12,17)$, we observed that the renal clearance of unbound pindolol was greater than the GFR, which indicates that pindolol is actively secreted by the kidneys. The data do not exclude the possibility that reabsorption, either passive or active, may also be occurring. Since $>95 \%$ of pindolol is positively charged at physiological $\mathrm{pH}$ values (18), it is possible that the organic cation transport system of the renal proximal tubule is involved in the secretion of pindolol from the kidney (19) and that this system is stereoselectively transporting the drug. Upon perusal of the literature, we did not find any information regarding the stereoselectivity of the organic cation transport system.

In this study, the mean difference in net clearance by secretion of $d$ - and $l$-pindolol was $\sim 30 \%$. Since pindolol has a relatively high therapeutic ratio (16), the clinical significance of these differences is probably unimportant. However, the study may have clinical implications for other organic bases that are renally eliminated and basic implications for the organic cation transport system of the renal proximal tubule.

In general, the renal clearance of the individual enantiomers was not dependent upon plasma concentration, time, or urine $\mathrm{pH}$. In three of the six subjects, there was a significant correlation of renal clearance vs. urine flow, which suggests that the enantiomers are reabsorbed by passive diffusion. In addition, in two or three subjects, the fractional renal clearance tended to be larger when plasma concentrations were higher. Balant et al. (20) also observed that in subjects who had received a single oral dose of pindolol, the renal clearance of racemic pindolol was greater at early times when plasma concentrations were higher. These transiently greater renal clearances may be explained by early arterial-venous differences in drug concentration or by concentration-dependent reabsorption processes in the kidney.

In addition to studying directly the renal clearance of the enantiomers of pindolol, we also examined stereoselective differences in other pharmacokinetic properties. We observed that

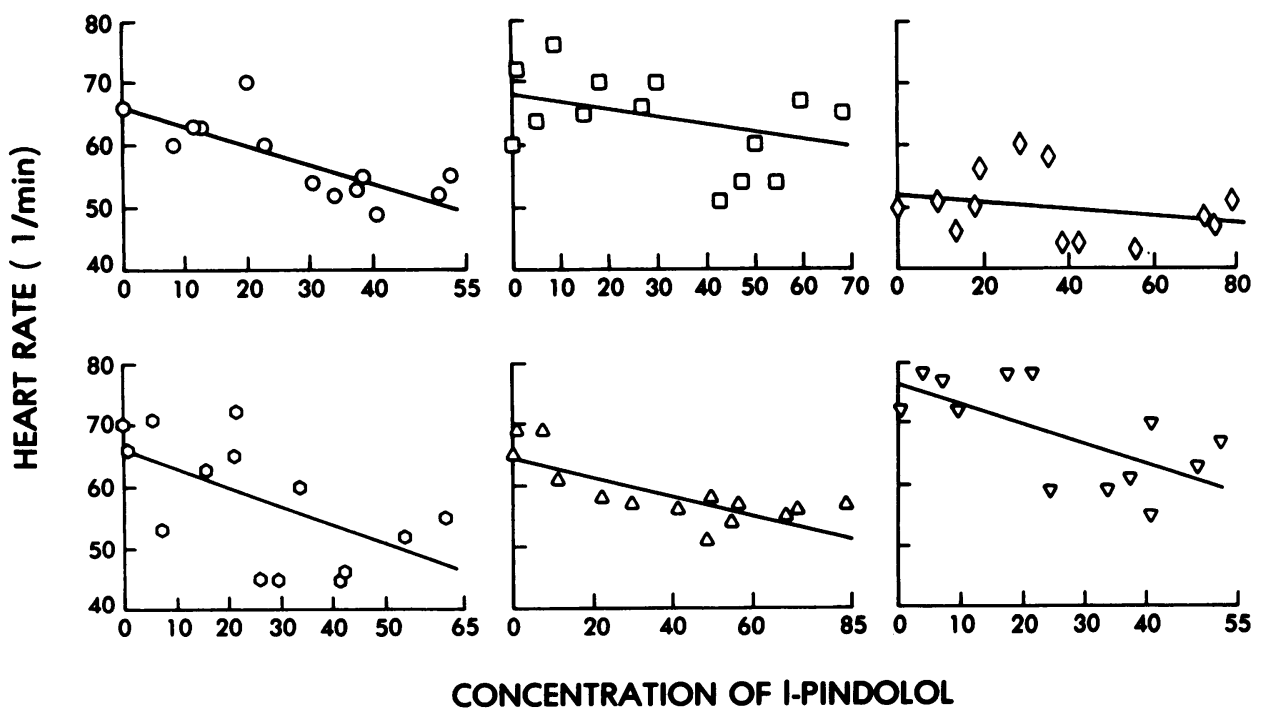

Figure 4. Heart rate vs. plasma concentration of $l$-pindolol in each subject. The lines were obtained by linear least-squares regression analysis. The slopes were significantly different from zero in subjects represented by the circles, hexagons, triangles, and inverted triangles $(P<0.05)$. 


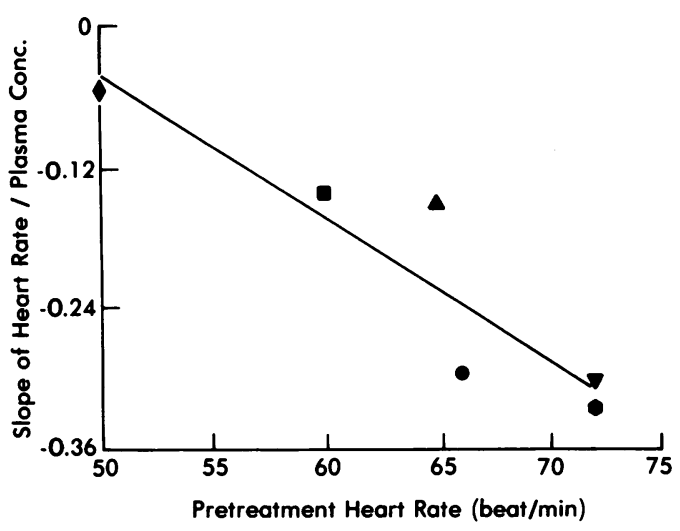

Figure 5. Slopes of a line describing the dependence of the heart rate on plasma concentration in each individual subject plotted against the pretreatment heart rate. The line represents the best fit obtained by linear least-squares regression analysis. The correlation coefficient was $-0.917(P<0.01)$.

the AUC and $\mathrm{Ae}^{\infty}$ of $l$-pindolol were greater than those of $d$ pindolol. Because AUC and $\mathrm{Ae}^{\infty}$ after oral administration are determined by both bioavailability and total body clearance, these data suggest that the bioavailability of $l$-pindolol is higher or that the total body clearance is lower than that of $d$-pindolol. Since the total body clearance is the sum of renal and nonrenal clearance and we observed that the renal clearance of $l$-pindolol was greater than that of $d$-pindolol, these data suggest that $l$ pindolol may be cleared more slowly by nonrenal routes such as metabolism than the corresponding $d$-enantiomer. The longer half-life of $l$-pindolol also supports the notion that the total body clearance of $l$-pindolol is lower than that of $d$-pindolol and therefore $l$-pindolol is eliminated more slowly by metabolism. Alternatively, the longer half-life of $l$-pindolol may be a result of a larger apparent volume of distribution; however, because the drug was orally administered, the volume of distribution could not be ascertained. The simplest explanation for the observed stereoselective differences in half-life, $\mathrm{AUC}, \mathrm{Ae}^{\infty}$, and renal clearance is that there is both stereoselective metabolism and renal excretion, with $l$-pindolol being excreted more rapidly by the kidney and cleared more slowly by nonrenal mechanisms than the corresponding $d$-enantiomer.

Several studies $(21,22)$ have demonstrated that despite its intrinsic sympathomimetic activity, therapeutic doses of pindolol may decrease the resting heart rate. However, there have been no studies demonstrating a relationship between heart rate and plasma concentration of pindolol. In fact, Carruthers and TwumBarima (21) administered cumulative oral doses of pindolol ranging between 2.5 and $57.5 \mathrm{mg}$ to normal volunteers and observed that the effect of pindolol on the resting heart rate was independent of dose and therefore, by inference, independent of plasma concentration. In contrast to their study, the results of this study demonstrated that the resting heart rate was related to the plasma concentration of pindolol. In all volunteers, the slope of the line relating heart rate to plasma concentration was negative, and in four of the six subjects, the slope was significantly different from zero. In accordance with other investigations $(21,23)$, we have observed that the effect of pindolol on the resting heart rate is largely determined by an individual's sympathetic tone. However, our results differ from previous studies because we related the resting heart rate to the plasma concentration of pindolol.
We observed a significant correlation between the volunteers' pretreatment heart rates and the slopes of the heart rate vs. the plasma concentration curves. Volunteers with greater resting heart rates had a greater sensitivity to pindolol, as expressed by a more negative slope of the line defining the relationship between heart rate and plasma concentration (Fig. 5).

In summary, this study has demonstrated that pindolol is eliminated from the kidneys in humans by stereoselective mechanisms. Since binding of pindolol to plasma proteins was not stereoselective, renal handling mechanisms are implicated. A discussion has been presented supporting the notion that either stereoselective renal transport or stereoselective renal metabolism is occurring. In addition to stereoselective renal elimination, the data suggest that pindolol is also eliminated by stereoselective metabolism. A significant relationship between the plasma concentration of pindolol and the resting heart rate has been observed. The slope of this relationship appears to be correlated and perhaps dependent upon the pretreatment resting heart rate, which suggests that sympathetic tone determines the negative chronotropic response to pindolol.

\section{Acknowledgments}

We are grateful to Dr. Roger Williams for his advice on the clinical study and to Andrea Mazel for typing the manuscript.

This research was supported by grant GM31254 from the National Institutes of Health.

\section{References}

1. Barrett, A. M., and V. A. Cullum. 1968. The biological properties of the optical isomers of propranolol and their effects on cardiac arrhythmias. Br. J. Pharmacol. 34:43-55.

2. Silber, B., and S. Riegelman. 1980. Stereospecific assay of (-)and (+)-propranolol in human and dog plasma. J. Pharmacol. Exp. Ther. 215:643-648.

3. Silber, B., N. H. G. Holford, and S. Riegelman. 1982. Stereoselective disposition and glucuronidation of propranolol in humans. J. Pharm. Sci. 71:699-703.

4. Testa, B., and P. Jenner. 1980. A structural approach to selectivity in drug metabolism and disposition. In Concepts in Drug Metabolism. P. Jenner and B. Testa, editors. Marcel Dekker, Inc., New York and Basel. 75-143.

5. Lewis, R. J., W. F. Trager, K. K. Chan, A. Breckenridge, M. Orme, M. Rowland, and W. Schary. 1974. Warfarin. Stereochemical aspects of its metabolism and the interaction with phenylbutazone. J. Clin. Invest. 53:1607-1617.

6. Ross, C. R., and P. D. Holohan. 1983. Transport of organic anions and cations in isolated renal plasma membranes. Annu. Rev. Pharmacol. Toxicol. 23:65-85.

7. Golightly, L. K. 1982. Pindolol: a review of its pharmacology, pharmacokinetics, clinical uses, and adverse effects. Pharmacotherapy. 2:134-147.

8. Holford, N. H. G. 1982. DRUGFUN. In PROPHET Public Procedures. H. M. Perry, editor. National Institutes of Health, Bethesda, MD. 4:63-81.

9. Holford, N. H. G. 1982. MODELTEST. In PROPHET Public Procedures. H. M. Perry, editor. National Institutes of Health, Bethesda, MD. 4:139-145.

10. Gibaldi, M., and D. Perrier. 1982. Pharmacokinetics. Marcel Dekker, Inc., New York and Basel. 477 pp.

11. Clark, B. J., and A. Bertholet. 1983. Effects of pindolol on vascular smooth muscle. Gen. Pharmacol. 14:117-119. 
12. Meier, J. 1982. Pharmacokinetic comparison of pindolol with other beta-adrenoceptor blocking agents. Am. Heart J. 104:364-372.

13. Ohnhaus, E. E., E. Nüesch, J. Meier, and F. Kalberer. 1974. Pharmacokinetics of unlabelled and ${ }^{14} \mathrm{C}$-labelled pindolol in uraemic. Eur. J. Clin. Pharmacol. 7:25-29.

14. Øie, S., and G. Levy. 1975. Relationship between renal function and elimination kinetics of pindolol in man. Eur. J. Clin. Pharmacol. 9:115-116.

15. Ohnhaus, E. E., U. Münch, and J. Meier. 1982. Elimination of pindolol in liver disease. Eur. J. Clin. Pharmacol. 22:247-251.

16. Ohnhaus, E. E., H. Heidemann, J. Meier, and G. Maurer. 1982. Metabolism of pindolol in patients with renal failure. Eur. J. Clin. Pharmacol. 22:423-428.

17. Gugler, R., W. Herold, and H. J. Dengler. 1974. Pharmacokinetics of pindolol in man. Eur. J. Clin. Pharmacol. 7:17-24.
18. Lemaire, M., and J. P. Tillement. 1982. The binding characteristics of some adrenergic beta-receptor antagonists to human serum proteins. Biochem. Pharmacol. 31:359-365.

19. Rennick, B. 1981. Renal tubule transport of organic cations. Am. J. Physiol. 240:F83-F89.

20. Balant, L., K. Muir, P. Dayer, A. Gorgia, J. L. Eberlin, J. Estreicher, and J. Fabre. 1981. Simultaneous tubular excretion and reabsorption of pindolol in man. Eur. J. Clin. Pharmacol. 21:65-72.

21. Carruthers, S. G., and Y. Twum-Barima. 1982. Measurement of partial agonist activity of pindolol. Clin. Pharmacol. Ther. 30:581-586.

22. Aellig, W. H. 1977. Investigations with $\beta$-adrenoceptor blocking drugs in healthy volunteers. Acta. Med. Scand. 606(Suppl.):715-755.

23. Rosenthal, J., H. Kaiser, A. Raschig, and D. Welzel. 1979. Treatment of hypertension with a beta-adrenoceptor blocker. A multicentre trial with pindolol. Br. J. Clin. Pract. 33:165-174. 